\title{
Ruptured Echinococcus granulosus cysts in migrants: Is excessive antigen release causing false negative serology?
}

Dear Editor,

Recently, three female patients with a migratory background (Surinam, Afghanistan and Turkey) presented in our hospital with signs of an anaphylactic shock due to a spontaneous Echinococcus granulosus cyst rupture. Although the current literature states that serology for Cystic Echinococcosis (CE) should be positive in these patients $[1,2]$, serology was found to be negative in all three patients at presentation. This prompted us to review the literature, which resulted in a hypothesis that could explain the false negative serology in patients with anaphylaxis due to $\mathrm{CE}$.

\section{False negative serology in anaphylactic patients with cystic echinococcosis}

CE is a severe zoonotic disease caused by the cestode species Echinococcus granulosus and is mainly seen in patients originating from endemic areas such as the Mediterranean countries, China, South America and North and East Africa [2]. Patients develop large cysts filled with numerous protoscolices and hence Echinococcus antigens [2]. Upon rupture of these cysts, cyst contents are released and may cause anaphylaxis, which was recently experienced by three patients in our hospital. The cyst location in these patients was hepatic (twice) and pulmonic, as determined by imaging. Specific antibodies for CE were absent in all three CE patients in samples collected at the time of presentation. However, specific antibodies were present in samples taken $\geq 6$ days after admission (Fig. 1A). Because CE was already suspected, all three patients were treated with albendazole from the acute presentation onwards and they all made a full recovery. However, if the negative serology would have resulted in a wait and see policy, secondary CE could have developed, causing severe problems.

\subsection{Mechanism of false negative serology}

We postulate the following mechanism for the false negative serological results when patients are in an anaphylactic state (Fig. 1B). Massive antigen release and the subsequently formed antibody-antigen complexes are thought to evoke the anaphylactic reaction. Upon cyst rupture, antigens are abruptly and excessively released in the cyst environment, which will saturate all (or a substantial part of) the available specific antibodies if there is access to the circulation. The anaphylactic symptoms of the patients showed that in our cases this route was accessible. In serological assays the presence of specific antibodies is tested through their capability to bind to specific antigens. However, if the specific antibodies have already bound released antigens in the circulation of the patient, these specific antibodies are saturated and have become non-reactive in serological assays. As a consequence, a (false) negative test result will be obtained. The proposed mechanism is different from the "prozone effect", in which the excess of either antigens or antibodies in the assay results in decreased reactivity [3]. In our case the antibodies in the blood sample have bound antigen from the ruptured cyst, thereby rendering dilution, the normal method to curb the prozone effect, ineffective.

\section{Conclusions}

We proposed a mechanism that explains false-negative serology in patients with a ruptured Echinococcus cyst that are experiencing anaphylactic shock. Therefore, when initial serological results are negative and a ruptured CE cyst is suspected, we advise to repeat CE serology after at least a week before ruling out $\mathrm{CE}$ as the cause of the anaphylactic shock. 
A

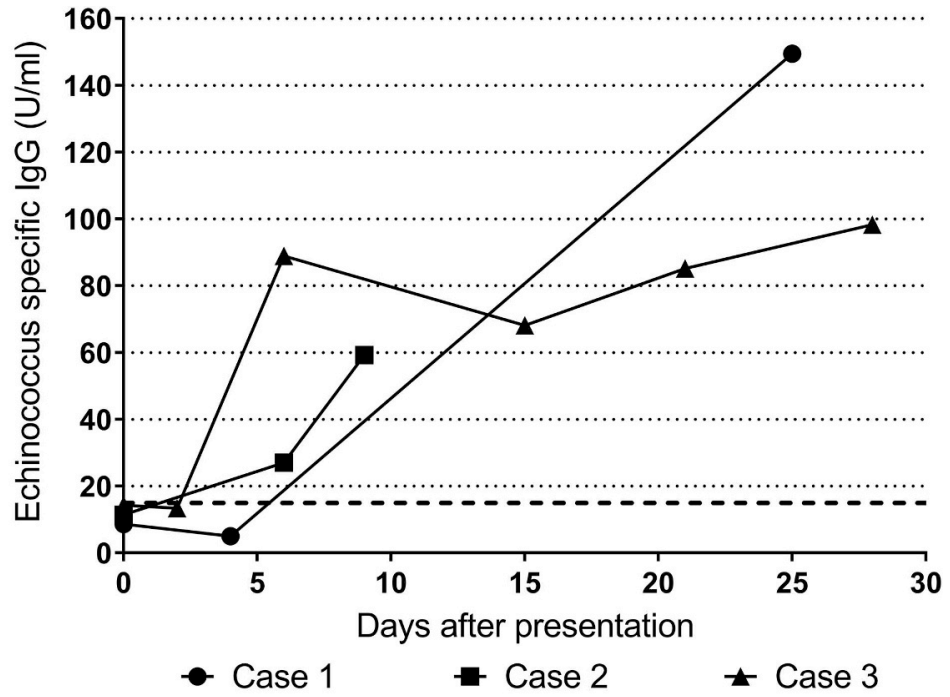

B

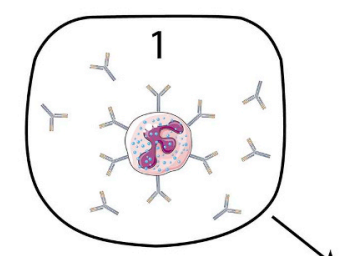

3

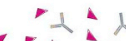

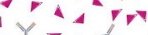

(i)

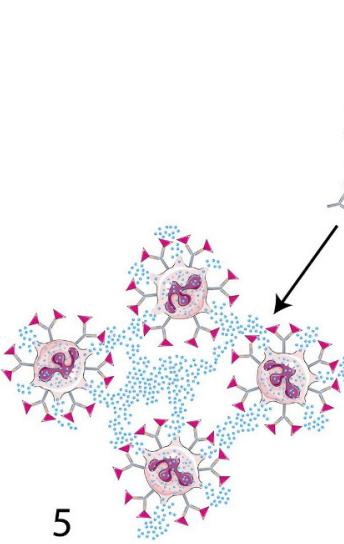

Fig. 1. Panel A: Echinococcus specific IgG levels over time of the three presented cases. The dashed line at $15 \mathrm{U} / \mathrm{ml}$ represents the cut-off point. E. granulosus specific antibodies were determined with the SERION ELISA classic Echinococcosis IgG test [4]. For two of the three patients the negative as well as the subsequent positive serological results were confirmed by two other methods; a commercially available Indirect Haem Agglutination assay (IHA, Fumouze) and an in-house developed ELISA. For one patient, no material was left for retrospective confirmation. Panel B: Proposed mechanism of false-negative serology results in serum collected at presentation of patients with an anaphylactic reaction. In a predisposed individual with high IgE (1), release of antigen through cystic echinococcosis cyst rupture (2) may result in abundant release of antigens into the bloodstream (3), which will bind to all available free and mast-cell bound IgE (4). This will result in release of inflammatory mediators by mast cells, causing anaphylaxis (5). Simultaneously, the antigen-antibody complexes will not bind to antigens provided in the serological assay, resulting in false negative results (6). 


\section{References}

[1] Murali MR, Uyeda JW, Tingpej B. Case records of the Massachusetts General Hospital. Case 2-2015. A 25-year-old man with abdominal pain, syncope, and hypotension. N Engl J Med 2015;372:265-73.

[2] Nunnari G, Pinzone MR, Gruttadauria S, Celesia BM, Madeddu G, Malaguarnera G, et al. Hepatic echinococcosis: clinical and therapeutic aspects. World J Gastroenterol 2012;18:1448-58.

[3] Butch AW. Dilution protocols for detection of hook effects/prozone phenomenon. Clin Chem 2000:46:1719-21.

[4] Sarink MJ, Koelewijn R, Slingerland BCGC, Tielens AGM, van Genderen PJJ, van Hellemond JJ. Performance of the commercially available SERION ELISA classic Echinococcus IgG test for the detection of cystic echinococcosis in clinical practice. J Helminthol 2019. (in press), PMID:29950187, 10.1017/S0022149X18000536.
Maarten J. Sarink, Perry J.J. van Genderen

Aloysius G.M. Tielens

Department of Medical Microbiology and Infectious Diseases, Erasmus MC University Medical Center, Dr. Molewaterplein 40, Rotterdam, the Netherlands

Department of Biochemistry and Cell Biology, Faculty of Veterinary Medicine, Utrecht University, Utrecht, the Netherlands

Jaap J. van Hellemond*

Department of Medical Microbiology and Infectious Diseases, Erasmus MC University Medical Center, Dr. Molewaterplein 40, Rotterdam, the Netherlands

E-mail address: j.vanhellemond@erasmusmc.nl.

${ }^{*}$ Corresponding author. Dept. Medical Microbiology \& Infectious Diseases, Erasmus MC University Medical Centre, Dr. Molewaterplein 40, P.O. box 2040, 3000, CA, Rotterdam, the Netherlands. 